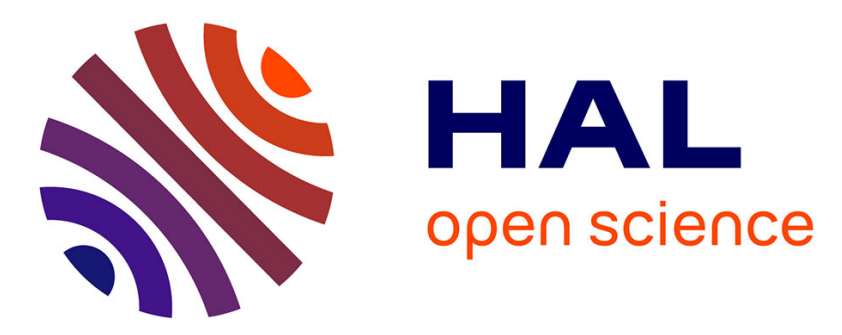

\title{
Experimental demonstration of an ultrafast all-optical bit-error indicating scheme
}

\author{
Christophe Finot, Julien Fatome
}

\section{To cite this version:}

Christophe Finot, Julien Fatome. Experimental demonstration of an ultrafast all-optical biterror indicating scheme. Microwave and Optical Technology Letters, 2011, 53 (2), pp.392-395. 10.1002/mop.25711 . hal-00521055

\section{HAL Id: hal-00521055 \\ https://hal.science/hal-00521055}

Submitted on 4 Dec 2010

HAL is a multi-disciplinary open access archive for the deposit and dissemination of scientific research documents, whether they are published or not. The documents may come from teaching and research institutions in France or abroad, or from public or private research centers.
L'archive ouverte pluridisciplinaire HAL, est destinée au dépôt et à la diffusion de documents scientifiques de niveau recherche, publiés ou non, émanant des établissements d'enseignement et de recherche français ou étrangers, des laboratoires publics ou privés. 


\title{
Experimental demonstration of an ultrafast all-optical bit-error indicating scheme
}

\author{
C. Finot * and J. Fatome \\ Laboratoire Interdisciplinaire CARNOT de Bourgogne, \\ UMR 5209 CNRS-Université de Bourgogne, \\ 9 Av. A. Savary, BP 47 870, 21078 DIJON Cedex, FRANCE \\ *christophe.finot@u-bourgogne.fr
}

\begin{abstract}
We experimentally demonstrate an all-optical bit error monitoring scheme based on the self-phase modulation occurring during the propagation in a highly nonlinear fiber followed by an optical bandpass filter. Numerical simulations are confirmed by experimental observations performed at a repetition rate of $40 \mathrm{~Gb} / \mathrm{s}$.
\end{abstract}

Key words : nonlinear optics, all-optical processing, self-phase modulation, bit-error indicating scheme 


\section{Introduction}

With the advent of high-speed optical transmissions (40 Gb/s and beyond) and the increasing complexity of photonic networks, it becomes more and more crucial to develop adequate means to suitably characterize the quality of the optical data stream. Therefore, optical performance monitoring has become a very active field [1] that has recently stimulated intense research in order to overcome the bandwidth limitations of the usual electronics. The main target is to better detect and control the various impairments (noise, dispersion fluctuations, polarization mode dispersion...) that negatively impact the quality of service. In this context, any optical device that can highlight in real time the pulses responsible for detection error is highly desirable for improved fault management $[2,3]$.

In this paper, we experimentally demonstrate such a device. More precisely, based on the idea initially suggested by Oda and Maruta [4] and the related numerical investigation done by Zhang et al. [3], we confirm that the nonlinear response of optical fibers combined with an adequate optical spectral filtering can provide an efficient way to discriminate well-defined '0' or ' 1 ' bits of information from potential errors. We will first describe and illustrate the experimental set-up and the principle of operation of the device that we have implemented. Proof-of-principle experiments carried out at $40 \mathrm{~Gb} / \mathrm{s}$ will fully confirm the behaviour predicted by numerical simulations.

\section{Experimental set-up}

The configuration we investigate is sketched in Fig. 1. The experimental setup relies exclusively on commercially available components commonly used 
for optical telecommunications. A low jitter fiber laser source is actively modelocked by a 10-GHz RF signal and delivers Fourier-transform-limited picosecond pulses (2 ps, such a temporal duration is compatible with 160 $\mathrm{Gb} / \mathrm{s}$ telecommunication). A $\times 4$ bit rate multiplier (BRM) is used to simulate the basic degraded $40 \mathrm{GHz}$ return-to-zero pulse sequence with various levels that will constitute the signal under test (SUT). The resulting signal is sent into an erbium-doped fiber amplifier (EDFA) with a moderate output average power. The amplified pulse train then propagates into a highly nonlinear fiber (fiber from ofs specialty photonics division). This fiber is 920 meter long and presents a low anomalous dispersion $(D=0.7 \mathrm{ps} / \mathrm{km} / \mathrm{nm})$ combined with a high value of nonlinearity $(10 / \mathrm{W} / \mathrm{km})$. The linear losses and dispersion slope are both reduced $\left(0.6 \mathrm{~dB} / \mathrm{km}\right.$ and $0.01 \mathrm{ps}^{2} / \mathrm{km} / \mathrm{nm}$, respectively). The nonlinearity of the fiber leads to an intensity-dependent spectral expansion of the pulses due to self-phase modulation $[4,5]$. At the output of the fiber, an optical bandpass filter (OBPF) made of a fiber Bragg grating associated with an optical circulator $(\mathrm{OC})$ is used to carve into the expanded spectrum. The central wavelength of this filter is the same as the original stream and its spectral width is $200 \mathrm{GHz}$. The resulting error bit indicating signal (EBIS) is analyzed by means of a usual photodetector combined with a high-speed digital sampling oscilloscope (50 GHz optical bandwidth).

\section{Principle of operation}

The key element in this set-up is the optical transfer function (TF) that links the input and output powers. According to the choice of parameters of the 
fiber and the filter, the TF shape may significantly vary [6]. Indeed, the architecture described in Fig. 1 is well-known in the context of optical regeneration $[6,7,8,9]$, in which the aim is not to facilitate the detection of errors but to limit output pulse train jitter and act as an all-optical power limiter : this requires a TF with a large plateau characterized by an inflexion point. Another application based on similar architecture has also been recently proposed and targets the all-optical magnification of amplitude jitter [10]. Our purpose is here very different from optical regeneration or optical magnification so that the requirements on the TF differ significantly. More precisely and as it can be seen in Fig. 2, the TF needs to exhibit a nonmonotonous behaviour characterized by two local minima (initial powers 0 and $12 \mathrm{~mW}$ ) having an output value close to zero. A maximum of the TF should be observed for powers near the middle of the two minima (around $7 \mathrm{~mW}$ in our case).

The principle of the device can then be easily understood as follows [3, 4] : by properly adjusting the peak power of the ' 1 ' bits so as to be close to the second minima of the TF, the pulses emerging from the bit error indicating scheme will have a very low output level. On the contrary, the pulses having a peak power that may lead to a detection error fall in the range neighbouring the maximum of the TF. Consequently, they will exhibit a high output level and will be straightforwardly detected.

Let us note that the experimental scheme that we have implemented could have been replaced by other well-documented fibered systems presenting similar non-monotonous TF. For example, alternatives based on Mamyshev-regenerator like schemes $(M R)[11,12,13,14]$ or on nonlinear 
optical loop mirror (NOLMs) [15] could be considered. Let us however point out that in the case of the MR which is based on a very similar physical principle (self-phase modulation in an optical fibre, but operating in the normal dispersion regime and with an offset filtering), the required input power will be significantly higher and the process may additionally induce some detrimental timing jitter as well as pulse-to-pulse interaction, which may complexify the detection process $[6,16,17]$. Regarding the use of a NOLM, such a solution will not be appropriate : contrary to the proposed scheme or to MR, NOLMs are sensitive to the instantaneous power and not to the peak-power of the pulse.

\section{Numerical simulations}

In order to test the viability of the proposed approach, we have run some preliminary numerical simulations. The device under investigation can be modelled by the well known nonlinear Schrödinger equation $[5,6]$ in such a way that the TF can be predicted. Fig. 2 highlights the remarkable agreement achieved between the numerical simulations (solid grey line) and the experimental results (black circles). Based on this accurate model, we can test the ability of the device to efficiently detect the potential errors. Therefore, a realistic sequence of 16 bits (return-to-zero format) is plotted in Fig. 3a. The SUT is made of Gaussian pulses and presents a finite extinction ratio (linked to a finite extinction ratio of an initial modulator or to the growth of ghost pulses during the transmission [18]), fluctuations in the ' 1 ' power as well as a potential error. After the nonlinear device (Fig. 3b), both ' 1 ' bits and ' 0 ' bits 
present a reduced level whereas the error pulse has a significantly enhanced level. More careful inspection of the EBIS reveals that the output ' 1 ' state exhibits a double peak structure, but as no relevant measurement is carried out on those pulses, this does not constitute any drawback.

\section{Experimental results}

In order to experimentally simulate a given sequence including an error, we have used the BRM and starting from a $10 \mathrm{GHz}$ pulse train, we have obtained a $40 \mathrm{GHz}$ pulse trains with various peak-power levels. Two sequences have been tested. The first sequence, plotted in Fig. 4a1, presents some undesirable pulses in the ' 0 ' bits slots, which level could potentially lead to detection errors. In Fig. 4a2, at the output of our device, the outcoming stream reveals that the errors become readily apparent. The level of the ' 1 ' state is indeed significantly reduced. The ability of the device to conveniently deal with low level pulses, typically ghost pulses [18], is clearly reflected in the second sequence (Fig. 4b1 and 4b2). As a consequence, only one judge threshold is necessary to analyze the EBIS and to identify the potential errors. Less importantly, one can also notice from the various experiments that, in the EBIS,' 1 ' bits are affected by a much higher jitter than the ' 0 ' bits, which is consistent with the regime of amplitude jitter magnification [10]. Moreover, the previously mentioned double peaked structure can be guessed. 


\section{Conclusion}

We have experimentally demonstrated an easy-to-implement and costeffective device able to discriminate the undesirable pulses that may potentially compromise the detection from the ' 0 ' and ' 1 ' pulses having a regular level. The proposed all-optical function benefits from the quasiinstantaneous response of the Kerr non-linearity of optical fibers. Our experimental results obtained at a repetition rate of $40 \mathrm{~Gb} / \mathrm{s}$ can therefore be in principle extended to any higher repetition rates $(100 \mathrm{~Gb} / \mathrm{s}$ or even 160 $\mathrm{Gb} / \mathrm{s}[7])$. Measurements have been made on a single channel configuration, but using a counter-propagating configuration [19] or using polarization multiplexing [14], one can enable to handle as much as four different wavelengths simultaneously.

\section{Acknowledgements:}

This research was supported by the Agence Nationale de la Recherche (FUTUR project - ANR-06-TCOM-016, PERSYST 2 project - ANR-07-TCOM014) and by the Conseil Regional de Bourgogne. 


\section{Figure captions:}

Fig. 1 Experimental set-up.

Fig. 2 Transfer function of the proposed device. Experimental results (black circles, results obtained at a repetition rate of $10 \mathrm{Gbps}$ ) are compared with the numerical predictions based on the nonlinear Schrödinger equation (solid grey line).

Fig. 3 Numerical simulations. The SUT containing a potential error (subplot a) is compared with the EBIS (subplot b).

Fig. 4 Experimental results. Input (subplots 1) and output (subplots 2) pulses are compared for two sequences (subplots $a$ and $b$ ). 
Figure 1

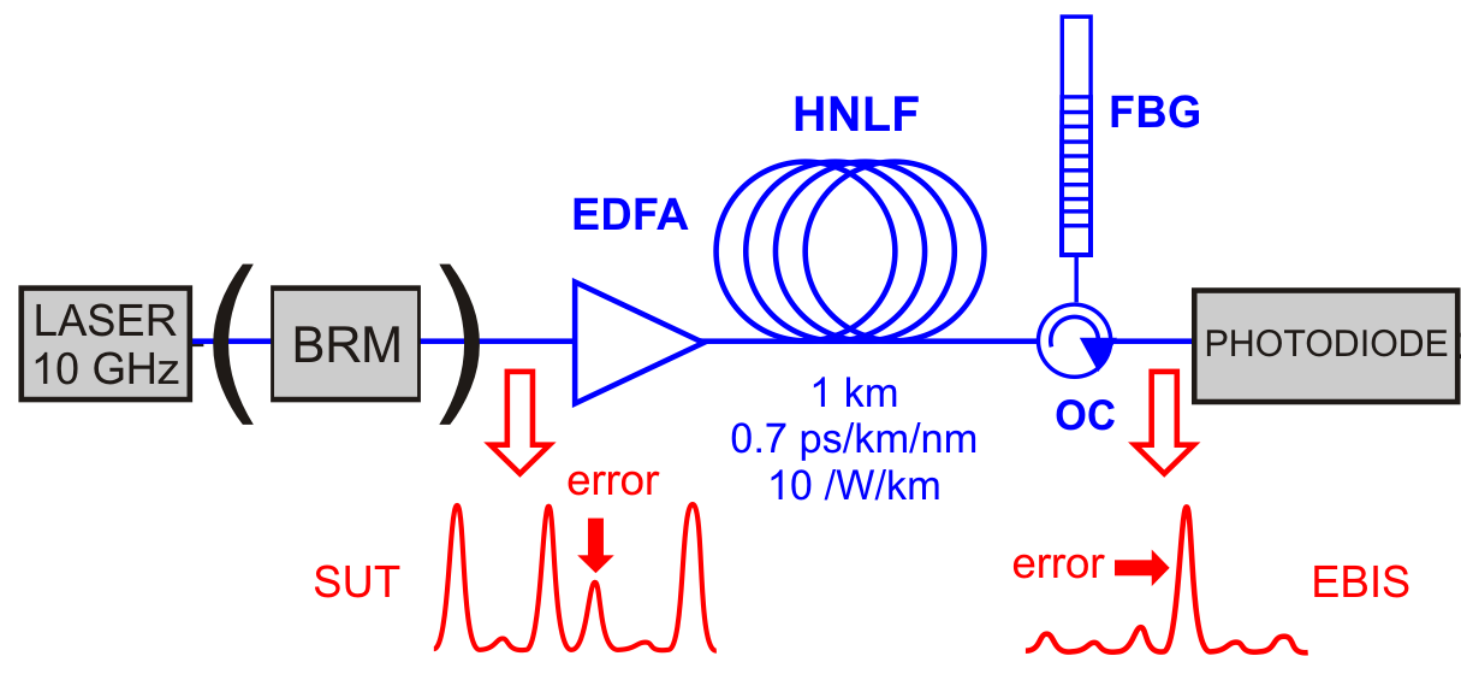


Figure 2

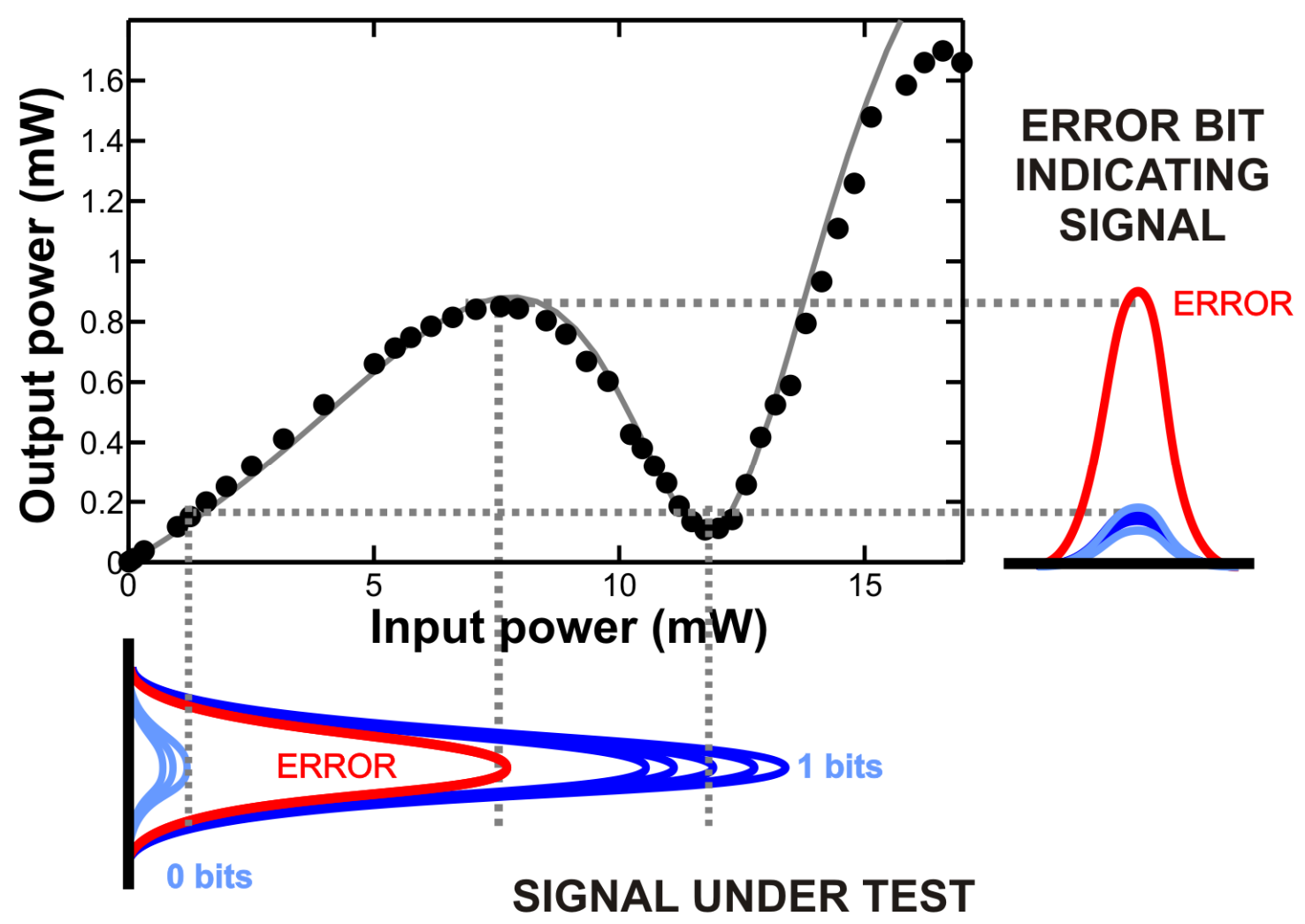


Figure 3

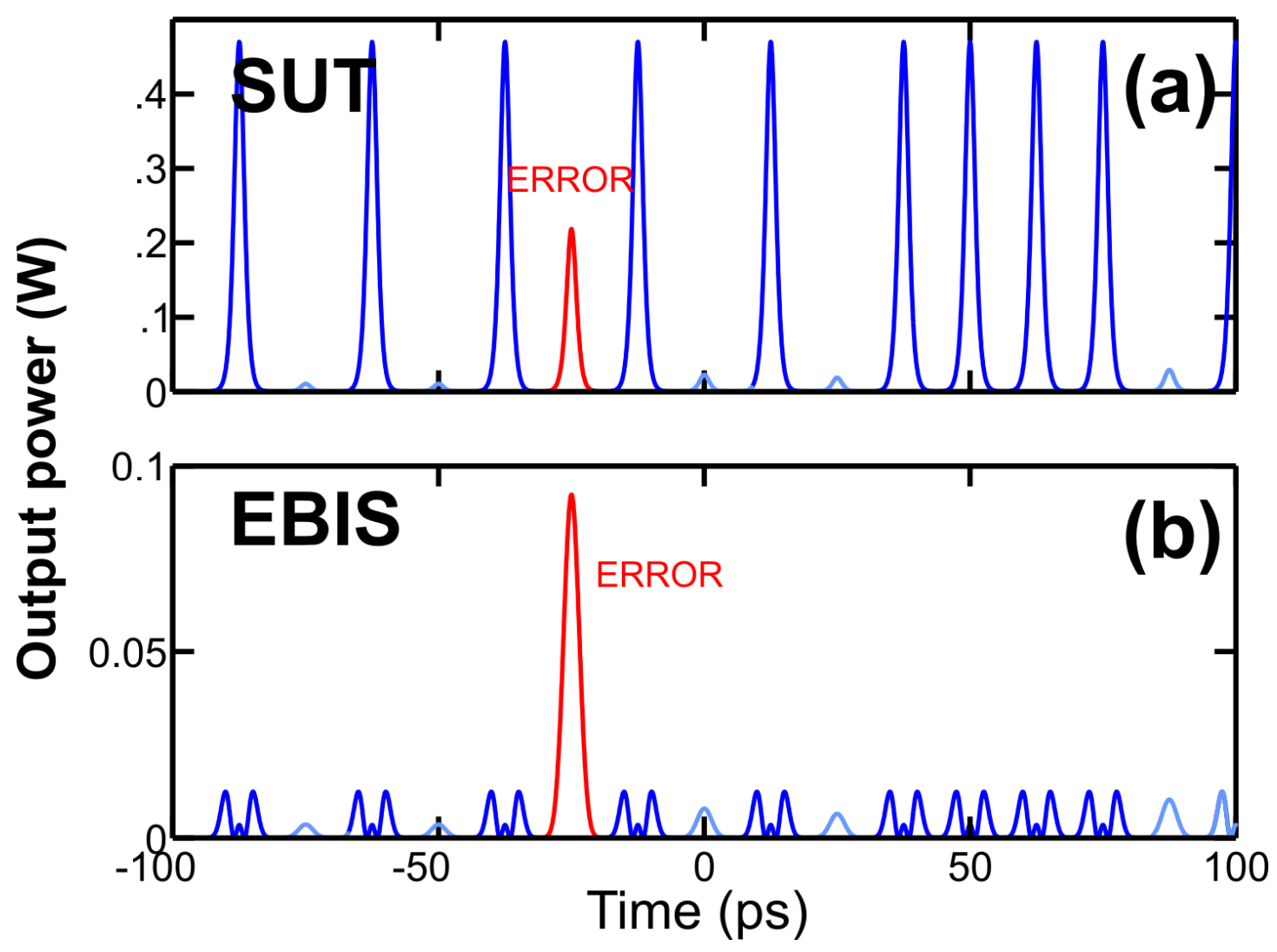


Figure 4

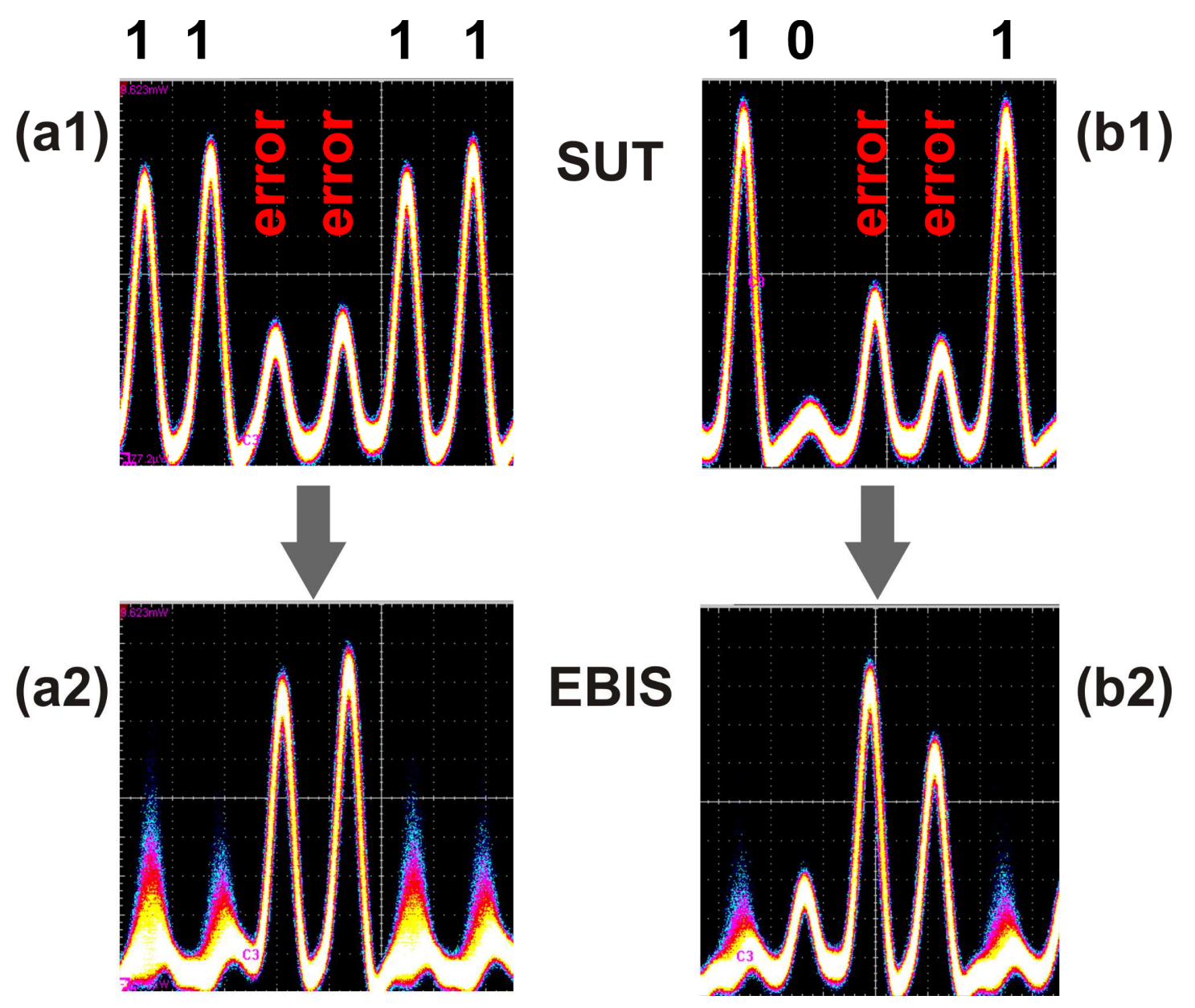




\section{References}

1. Z. Pan, C. Yu and A. E. Willner, Optical performance monitoring for the next generation optical communication networks, Opt. Fiber. Technol. 16 (2010), no. 1, 20-45.

2. L. Y. Chan, K. K. Qureshi, P. K. A. Wai, B. Moses, L. F. K. Lui, H. Y. Tam and M. S. Demokan, All-optical bit-error monitoring system using cascaded inverted wavelength converter and optical nor gate, IEEE Photon. Technol. Lett. 15 (2003), no. 4, 593-595.

3. Z. Zhang, X. Zhou, R. Liang, Z. Qin and Y. Liu, An all-optical bit-error indicating scheme based on self-phase modulation and filtering, Opt. Commun. 282 (2009), no. 15, 3163-3167.

4. S. Oda and A. Maruta, Two-bit all-optical analog-to-digital conversion by filtering broadened and split spectrum induced by soliton effect or self-phase modulation in fiber., IEEE J. Sel. Top. Quantum Electron. 12 (2006), no. 2, 307-314.

5. G. P. Agrawal, Nonlinear fiber optics, fourth edition, Academic Press, San Francisco, CA, 2006.

6. J. Fatome and C. Finot, Scaling guidelines of a soliton-based power limiter for 2r-optical regeneration applications, 11th International Conference on Transparent Optical Networks, IEEE, 2009, 1303-1306

7. T. Ohara, H. Takara, S. Kawanishi, T. Yamada and M. M. Fejer, 160 $\mathrm{gb} / \mathrm{s}$ all-optical limiter based on spectrally filtered optical solitons, IEEE Photon. Technol. Lett. 16 (2004), no. 10, 2311-2313.

8. M. Asobe, A. Hirano, Y. Miyamoto, K. Sato, K. Hagimoto and Y. Yamabayashi, Noise reduction of $20 \mathrm{gbit} / \mathrm{s}$ pulse train using spectrally filtered optical solitons, Electron. Lett. 34 (1998), no. 11, 1135-1136.

9. M. Gay, M. Costa e Silva, T. N. Nguyen, L. Bramerie, T. Chartier, M. Joindot, J. C. Simon, J. Fatome, C. Finot and J. L. Oudar, $170 \mathrm{gbit} / \mathrm{s}$ bit error rate assessment of regeneration using a saturable absorber and a nonlinear fiber based power limiter, IEEE Photon. Technol. Lett. 22 (2010), no. 3, 158-160.

10. J. Fatome and C. Finot, All-optical fiber-based amplitude jitter magnifier, 12th International Conference on Transparent Optical Networks, IEEE, 2010. 
11. P. V. Mamyshev, All-optical data regeneration based on self-phase modulation effect, European Conference on Optical Communication, ECOC'98, 1998, p. 475-476.

12. X. Zhang, X. M. Ren, N. Zhao, Y. Wang, L. Zheng and Y. Huang, Extinction ratio enhancement of self-phase modulation based all-optical regenerated signals in microstructuted fibers, Microw. Opt. Technol. Lett. 52 (2010), no. 2, 347-351.

13. Y. Yang and C. Lou, Experimental investigation of the influence of filters on regeneration performance in self-phase modulation based regenerator, Microw. Opt. Technol. Lett. 49 (2007), no. 1, 192-195.

14. T. Ohara, H. Takara, A. Hirano, K. Mori and S. Kawanishi, $40 \mathrm{gb} / \mathrm{s} \times 4$ channel all-optical multichannel limiter utilizing spectrally filtered optical solitons, IEEE Photon. Technol. Lett. 15 (2003), no. 5, 763-765.

15. S. Boscolo, S. K. Turitsyn and K. J. Blow, Nonlinear loop mirror-based all-optical signal processing in fiber-optic communications, Opt. Fiber. Technol. 14 (2008), no. 4, 299-316.

16. C. Finot, T. N. Nguyen, J. Fatome, T. Chartier, L. Bramerie, M. Gay, S. Pitois and J. C. Simon, Numerical study of an optical regenerator exploiting self-phase modulation and spectral offset filtering at 40 gbit/s, Opt. Commun. 281 (2008), 2252-2264.

17. L. Provost, C. Finot, K. Mukasa, P. Petropoulos and D. J. Richardson, Design scaling rules for 2r-optical self-phase modulation-based regenerators $2 r$ regeneration, Opt. Express 15 (2007), no. 8, 51005113.

18. P. V. Mamyshev and N. A. Mamysheva, Pulse-overlapped dispersionmanaged data transmission and intrachannel four-wave mixing, Opt. Lett. 24 (1999), no. 21, 1454-1546.

19. L. Provost, F. Parmigiani, C. Finot, K. Mukasa, P. Petropoulos and D. J. Richardson, Analysis of a two-channel $2 r$ all-optical regenerator based on a counter-propagating configuration, Opt. Express 16 (2008), no. 3, 2264-2275. 